\title{
NEAR-INFINITY CONCENTRATED NORMS AND THE FIXED POINT PROPERTY FOR NONEXPANSIVE MAPS ON CLOSED, BOUNDED, CONVEX SETS
}

\author{
F.E. CASTILLO-SÁNTOS, P.N. DOWLING, H. FETTER, M. JAPÓN, C.J. LENNARD, \\ B. SIMS, B. TURETT
}

\begin{abstract}
In this paper we define the concept of a near-infinity concentrated norm on a Banach space $X$ with a boundedly complete Schauder basis. When $\|\cdot\|$ is such a norm, we prove that $(X,\|\cdot\|)$ has the fixed point property (FPP); that is, every nonexpansive self-mapping defined on a closed, bounded, convex subset has a fixed point. In particular, P.K. Lin's norm in $\ell_{1}$ [14] and the norm $\nu_{p}(\cdot)$ (with $p=\left(p_{n}\right)$ and $\lim _{n} p_{n}=1$ ) introduced in [3] are examples of near-infinity concentrated norms. When $\nu_{p}(\cdot)$ is equivalent to the $\ell_{1}$-norm, it was an open problem as to whether $\left(\ell_{1}, \nu_{p}(\cdot)\right)$ had the FPP. We prove that the norm $\nu_{p}(\cdot)$ always generates a nonreflexive Banach space $X=\mathbb{R} \oplus_{p_{1}}\left(\mathbb{R} \oplus_{p_{2}}\left(\mathbb{R} \oplus_{p_{3}} \ldots\right)\right)$ satisfying the FPP, regardless of whether $\nu_{p}(\cdot)$ is equivalent to the $\ell_{1}$-norm. We also obtain some stability results.
\end{abstract}

\section{Introduction and Preliminaries}

Let $(X,\|\cdot\|)$ be a Banach space and $C$ a subset of $X$. A mapping $T: C \rightarrow C$ is said to be nonexpansive if $\|T x-T y\| \leq\|x-y\|$ for every $x, y \in C$. The Banach space $X$ endowed with the norm $\|\cdot\|$ has the fixed point property (FPP) if every nonexpansive mapping defined from a closed bounded convex subset $C$ of $X$ into itself has a fixed point. This property is not preserved by isomorphism, that is, it strongly depends on the underlying norm [14] . There is a wide literature relating geometric properties of reflexive Banach spaces with the fulfilment of the fixed point property (see, for instance, the monographs [9], [13] and the references therein).

The Banach space $\ell_{1}$ endowed with its standard norm $\|\cdot\|_{1}$ is a classical example of a nonreflexive Banach space that fails to have the FPP. It is possible to "perturb" this $\left(\ell_{1},\|\cdot\|_{1}\right)$-example to obtain other Banach spaces that fail to have the FPP. One such class of Banach spaces are those that contain asymptotically isometric copies of $\ell_{1}$.

Recall that a Banach space $(X,\|\cdot\|)$ contains an asymptotically isometric copy (a.i.c.) of $\ell_{1}$ if there are a sequence $\left(x_{n}\right) \subset X$ and a decreasing sequence $\left(\epsilon_{n}\right) \subset(0,1)$ with $\lim _{n} \epsilon_{n}=0$ such that

$$
\sum_{n=1}^{\infty}\left(1-\epsilon_{n}\right)\left|t_{n}\right| \leq\left\|\sum_{n=1}^{\infty} t_{n} x_{n}\right\| \leq \sum_{n=1}^{\infty}\left|t_{n}\right|
$$

1991 Mathematics Subject Classification. 46B03, 47H09, 47H10.

Key words and phrases. fixed point property; nonexpansive mappings; renorming theory.

The third author is partially funded by CONACYT grants 243722 and 613207 . The fourth author is partially supported by MCIN, Grant MTM2015-65242-C2-1-P and Andalusian Regional Government Grant FQM-127. She is also grateful to the Department of Mathematics of the University of Pittsburgh for its support while preparing this manuscript. The fifth author thanks the University of Newcastle for its financial support during part of the preparation of this paper; and Brailey, Gail and the Mathematics Department for their hospitality. 
for every $\left(t_{n}\right) \in \ell_{1}$. It was proved in [4] that if a Banach space contains an a.i.c. of $\ell_{1}$ then it fails to have the FPP. It turns out that there exist equivalent norms on $\ell_{1}$ which fail to contain an a.i.c. of $\ell_{1}$. Let us state some examples:

- The so-called P.K. Lin norm, defined as

$$
\||x|\|_{L}:=\sup _{k \geq 1} \gamma_{k} \sum_{n=k}^{\infty}|x(n)| ; \quad x=\sum_{n=1}^{\infty} x(n) e_{n}
$$

where $\left(\gamma_{k}\right)$ is a nondecreasing sequence in $(0,1)$ with $\lim _{k} \gamma_{k}=1$. In [5] it was proved that $\left(\ell_{1},\||\cdot|\|_{L}\right)$ fails to contain an a.i.c. of $\ell_{1}$. Later on, P.K. Lin [14] proved that $\left(\ell_{1},\left.|||\cdot|\right|_{L}\right)$ has the FPP for $\gamma_{k}:=\frac{8^{k}}{1+8^{k}}$. This condition was extended to every sequence $\left(\gamma_{k}\right)$ with $\lim _{k} \gamma_{k}=1$ (see [7] and [11]). P. K. Lin's result opened new avenues of research in the fixed point theory of nonexpansive mappings, since he settled negatively the long-standing open question: "Does the fixed point property imply reflexivity?" Since then, many other articles have appeared obtaining sufficient conditions that imply the FPP for equivalent norms on $\ell_{1}$ (see for instance $[2,6,7,8,10$, $11,12,15])$.

- Fix a nonincreasing sequence $p=\left(p_{n}\right)_{n} \subset(1,+\infty)$ with $\lim _{n} p_{n}=1$. In the sequence space $c_{00}$ of all real sequences with finitely many non-null coordinates, we define the norm $\nu_{p}(x)=\lim _{n} \nu_{n}(p, x)$ where

$$
\nu_{1}(p, x):=\left|x_{1}\right|, \quad \nu_{n+1}(p, x):=\left(\left|x_{1}\right|^{p_{1}}+\nu_{n}(S p, S x)^{p_{1}}\right)^{1 / p_{1}},
$$

with $x=\left(x_{1}, x_{2}, \ldots\right)$ and $S z:=\left(z_{2}, z_{3}, \ldots\right)$ when $z=\left(z_{1}, z_{2}, \ldots\right)$. The completion of $c_{00}$ with the $\nu_{p}(\cdot)$ norm gives us a Banach space $X$ with a boundedly complete Schauder basis $\left(e_{n}\right)$. Also, $X$ is the set of all real sequences $x=\left(x_{n}\right)$ for which $\nu_{p}(x):=\sup _{n} \nu_{n}(p, x)=\lim _{n} \nu_{n}(p, x)<\infty$; which we summarize by writing $X=\mathbb{R} \oplus_{p_{1}}\left(\mathbb{R} \oplus_{p_{2}}\left(\mathbb{R} \oplus_{p_{3}} \ldots\right)\right)$.

Let $q=\left(q_{n}\right)$ be the sequence satisfying $\frac{1}{p_{n}}+\frac{1}{q_{n}}=1$ for every $n \in \mathbb{N}$. Whenever the sequence $\left(p_{n}\right)$ converges to 1 quickly enough, the norm $\nu_{p}(\cdot)$ provides an equivalent norm in $\ell_{1}$; that is, $\left(X, \nu_{p}(\cdot)\right)$ and $\ell_{1}$ are isomorphic Banach spaces. In fact, it was proved in [3, Proposition 1] that $\nu_{p}(\cdot)$ is equivalent to the $\ell_{1}$ norm if and only if there exists some $\delta>0$ so that $q_{n} \geq \delta \log n$ for all $n \in \mathbb{N}$. It is also known that $\left(\ell_{1}, \nu_{p}(\cdot)\right)$ fails to contain asymptotically isometric copies of $\ell_{1}[3$, Theorem 1]. However, unlike P.K. Lin's norm, it was unknown whether $\ell_{1}$ with the norm $\nu_{p}(\cdot)$ had the fixed point property.

In what follows, we enlarge the class of norms on $\ell_{1}$ satisfying the FPP and we include, as a particular case, the norm $\nu_{p}(\cdot)$ defined in [3]. We will extend our result to a more general framework. For instance, we will prove the fulfilment of the FPP for $\left(X, \nu_{p}(\cdot)\right)$ even when this norm fails to be an $\ell_{1}$-norm.

Furthermore, we obtain stability of the fixed point property for certain norms along rays emanating from near-infinity concentrated norms.

\section{NEAR-INFINITY CONCENTRATED NORMS AND THE FPP}

Throughout this paper, let $X$ denote a Banach space with a Schauder basis $\left\{e_{n}\right\}_{n}$. Given $x=\sum_{n=1}^{\infty} x(n) e_{n} \in X$, we denote by $\operatorname{supp}(x)=\{n \in \mathbb{N}: x(n) \neq 0\}$, $Q_{k}(x)=\sum_{n=k}^{\infty} x(n) e_{n}$ and $P_{k}(x)=\sum_{n=1}^{k-1} x(n) e_{n}\left(P_{1}=0\right)$. The basis is said to be premonotone for the norm $\|\cdot\|$ when $\left\|Q_{k}\right\| \leq 1$ for every $k \in \mathbb{N}$. 
Given $k \in \mathbb{N}$ and $x \in X$, we write $k \leq x$ whenever $k \leq \min \{\operatorname{supp}(x)\}$ and $k<x$ whenever $k<\min \{\operatorname{supp}(x)\}$. We say that $\left(y_{n}\right)$ is a block basic sequence for $\left\{e_{n}\right\}_{n}$ if it is bounded and there exist positive integers $p_{1} \leq q_{1}<p_{2} \leq q_{2}<\ldots$ such that $y_{n}$ belongs to the span of $\left\{e_{p_{n}}, \cdots, e_{q_{n}}\right\}$ for every $n \in \mathbb{N}$.

The Schauder basis is said to be boundedly complete if $\sup _{n}\left\|\sum_{i=1}^{n} t_{i} e_{i}\right\|<+\infty$ implies that $\sum_{i=1}^{\infty} t_{i} e_{i} \in X$. When the Schauder basis $\left(e_{n}\right)$ is boundedly complete, the Banach space $X$ is isomorphic to a dual space $Z^{*}$, where $Z$ is the closed subspace spanned by the biorthogonal functionals $\left(e_{n}^{*}\right)$ in $X^{*}$. In this case, we can consider in $X$ the weak* topology $\sigma(X, Z)$, for which the convergence coincides with the coordinate-to-coordinate convergence for norm-bounded sequences. Moreover, the closed unit ball is $\sigma(X, Z)$-sequentially compact and therefore every bounded sequence in $X$ has a subsequence which converges coordinatewise (see for instance Theorem 3.2.10 in [1]). In what follows the weak* topology always refers to the $\sigma(X, Z)$ topology for Banach spaces with boundedly complete Schauder basis. In the case where $X=\ell_{1}$ endowed with the standard Schauder basis, this $w^{*}$-topology coincides with the $\sigma\left(\ell_{1}, c_{0}\right)$ topology.

Definition 2.1. [2] A norm ||$\cdot|| \mid$ on a Banach space $X$ with a Schauder basis $\left\{e_{n}\right\}$ is said to be a sequentially separating norm if for every $\epsilon>0$ there exists some $k \in \mathbb{N}$ such that

$$
\left\||| x||\left|+\limsup _{n}\right||| x_{n}\right\|\left|\leq(1+\epsilon) \limsup _{n}\left\|\left|x+x_{n}\right|\right\|\right.
$$

whenever $k \leq x$ and $\left(x_{n}\right)_{n}$ is a block basic sequence of $\left\{e_{n}\right\}_{n}$ in $X$.

Definition 2.2. Let $X$ be a Banach space with a Schauder basis $\left\{e_{n}\right\}_{n}$ and let \|\|$\cdot \|$ be a norm on $X$. This norm is called near-infinity concentrated (n.i.c.) if it has the following properties:

(1) It is a sequentially separating norm.

(2) It is premonotone.

(3) There exist $R_{0}>5$ and $M \in[0,1)$ such that for every $k \in \mathbb{N}$, there exists a function $F_{k}:(0,+\infty) \rightarrow[0,+\infty)$ satisfying the following conditions:

(a) $\lim _{\lambda \rightarrow 0^{+}} \frac{F_{k}(\lambda)}{\lambda} \leq \frac{M}{R_{0}}$.

(b) For every bounded pointwise-null sequence $\left(x_{n}\right)$ with $\liminf \operatorname{in}_{n}\left\|\left|x_{n}\right|\right\| \geq$ 1 , for all $\lambda \in(0,+\infty)$, and for every $z \in X$ with $Q_{k}(z)=0$ and $\||z|\| \leq R_{0}$

$$
\limsup _{n}\left\|\left|x_{n}+\lambda z\left\|\left|\leq \limsup _{n}\left\|\mid x_{n}\right\|\left\|+F_{k}(\lambda)\right\|\|z\| \| .\right.\right.\right.\right.
$$

Remark 2.3. Observe that Property (3) can be re-written as: There exists $K \geq 0$ such that for every $k \in \mathbb{N}$, there exists a function $F_{k}:(0,+\infty) \rightarrow[0,+\infty)$ satisfying (a)' and (b); where condition (a)' is: $\lim _{\lambda \rightarrow 0^{+}} \frac{F_{k}(\lambda)}{\lambda} \leq K<\frac{1}{5}$.

Given $K$, we may take $M:=1-\frac{1-5 K}{K+1}=\frac{6 K}{K+1}$ and $R_{0}:=5+\frac{1-5 K}{K+1}=\frac{6}{K+1}$.

Note that if ||$\cdot \mid \|$ is an equivalent norm on $\ell_{1}$ satisfying

$$
a_{k}\left\|Q_{k}(x)\right\|_{1} \leq\|\| Q_{k}(x)\|\| b_{k}\left\|Q_{k}(x)\right\|_{1} \text {, for all } x \in \ell_{1},
$$


for every $k \in \mathbb{N}$, with $0<a_{k} \leq b_{k}$ and $\lim _{k} b_{k} / a_{k}=1$, then it is clear that ||$\cdot \mid \|$ is a sequentially separating norm. Nevertheless, there exist some equivalent norms on $\ell_{1}$ which do not satisify this condition but they are still sequentially separating $[2$, Example 3.2]. Furthermore, there exist Banach spaces with sequentially separating norms that are not isomorphic to $\ell_{1}$, although the existence of such a norm implies that the Banach space $X$ is "similar" to $\ell_{1}$, in the sense that it has the Schur property, and so is hereditarily $\ell_{1}[2$, Corollary 7.4]. Recall that a Banach space $X$ is hereditarily $\ell_{1}$ if each infinite dimensional closed subspace of $X$ contains a further subspace isomorphic to $\ell_{1}$. This implies, in particular, that if a Banach space with an unconditional Schauder basis has a sequentially separating norm, then the basis is boundedly complete, since otherwise $X$ would contain an isomorphic copy of $c_{0}$ (see for instance [1, Theorem 3.3.2]).

Also note that in Definition 2.2, Property $(3)(\mathrm{b})$, if $\left(x_{n}\right)$ is an arbitrary sequence of "bump functions sliding towards infinity", each with their ||| |||-norm asymptotically no less than 1 , then

$$
\frac{\lim \sup _{n}||\left|x_{n}+\lambda z\right|||-\lim \sup _{n}||\left|x_{n}\right| \|}{\lambda}
$$

is smaller than one would expect from just the triangle inequality: for all $z=P_{k}(z)$ with ||$|z||| \leq R_{0}$, for all $\lambda$ positive and very small, the "upper asymptotic value" of the norm of $x_{n}$ is changed less than expected when we perturb each, $x_{n}$ by $\lambda z$, since $F_{k}(\lambda) R_{0} / \lambda$ is approximately bounded by $M<1$. In this sense, $|\|\cdot \mid\|$ is "nearinfinity concentrated". Moreover, this third property prevents $X$ from containing an asymptotically isometric copy of $\ell_{1}$, which we will now prove.

Lemma 2.4. Let $X$ be a Banach space with a boundedly complete Schauder basis. If $\|\cdot\| \|$ is an equivalent norm in $X$ satisfying property (3) in Definition 2.2, then $(X, \||\cdot| \mid)$ fails to have an a.i.c. of $\ell_{1}$.

Proof. Assume to the contrary that there exists a basic sequence $\left(x_{n}\right)$ in $X$ generating an a.i.c. of $\ell_{1}$, that is, there is a decreasing sequence $\left(\epsilon_{n}\right) \subset(0,1)$ with $\lim _{n} \epsilon_{n}=0$ such that

$$
\sum_{n=1}^{\infty}\left(1-\epsilon_{n}\right)\left|t_{n}\right| \leq\left\|\left|\sum_{n=1}^{\infty} t_{n} x_{n} \| \leq \sum_{n=1}^{\infty}\right| t_{n} \mid .\right.
$$

By extracting a subsequence, we can assume that $\left(x_{n}\right)$ is $w^{*}$-convergent and, by replacing $\left(x_{n}\right)$ by $\left.\left(\left(x_{2 n}-x_{2 n-1}\right) / 2\right)\right)$, that it is $w^{*}$-convergent to the null vector. Finally, using the sliding hump method and the fact that asymptotically isometric copies are stable by adding norm-null sequences, we can assume that the sequence $\left(x_{n}\right)$ generating the a.i.c. of $\ell_{1}$ is a disjointly supported $w^{*}$-null sequence.

Take $R_{0}>5$ and $M \in[0,1)$ as in (3) of Definition 2.2. By omitting the first few terms of the sequence $\left(x_{n}\right)_{n}$, we can also assume that $\epsilon_{1}<\left(R_{0}-M\right) / R_{0}$.

From the previous inequalities $\left\|\left|x_{n}\right|\right\| \leq 1$ for every $n \in \mathbb{N}$ and $\lim _{n}\left\|\left|x_{n}\right|\right\|=1$. Let $k:=1+\max \left\{\operatorname{supp}\left(x_{1}\right)\right\}$. Since \|\|$\cdot \mid \|$ satisfies property $(3)$ of a near-infinity concentrated norm, there exists a function $F_{k}(\lambda)$ such that $\lim _{\lambda \rightarrow 0^{+}} F_{k}(\lambda) / \lambda \leq \frac{M}{R_{0}}$, and for every $\lambda>0$

$$
\limsup _{n}\left|\left\|x_{n}+\lambda R_{0} x_{1}\right\|\right| \leq \limsup _{n}||\left|x_{n}\left\|\left|+F_{k}(\lambda) R_{0}\right|\right\| x_{1} \|\right| \leq 1+F_{k}(\lambda) R_{0} .
$$

On the other hand, for every $n \geq 2$,

$$
1-\epsilon_{n}+\lambda R_{0}\left(1-\epsilon_{1}\right) \leq\left\|\mid x_{n}+\lambda R_{0} x_{1}\right\| \|
$$

Letting $n$ tend to infinity, we see that

$$
1+\lambda R_{0}\left(1-\epsilon_{1}\right) \leq 1+F_{k}(\lambda) R_{0}
$$


and so $\lambda\left(1-\epsilon_{1}\right) \leq F_{k}(\lambda)$ for every $\lambda>0$. Letting $\lambda \rightarrow 0$, we get that $(1-$ $\left.\epsilon_{1}\right) \leq \lim _{\lambda \rightarrow 0^{+}} \frac{F_{k}(\lambda)}{\lambda} \leq \frac{M}{R_{0}}$, which implies that $R_{0}\left(1-\epsilon_{1}\right) \leq M$, and this is a contradiction.

Before stating our main result, we recall some standard arguments used to prove the FPP (see for instance [14] or [11]):

Let $C$ be a closed bounded convex subset of a Banach space $(X,\|\cdot\|)$ and $T: C \rightarrow C$ be a nonexpansive mapping. Using Banach's Contraction Mapping Theorem, we can always find a sequence $\left(x_{n}\right) \subset C$ such that $\lim _{n}\left\|x_{n}-T x_{n}\right\|=0$. Such sequences are called approximate fixed point sequences (a.f.p.s.). In fact, if $\left(x_{n}\right)$ is an a.f.p.s. and $r>0$, the set

$$
\left\{x \in C: \limsup _{n}\left\|x_{n}-x\right\| \leq r\right\}
$$

is either empty, or a non-empty closed convex $T$-invariant subset of $C$, in which we can find new approximate fixed point sequences.

In a dual Banach space $X$ with separable predual $Y$, every a.f.p.s. has a subsequence which is $w^{*}$-convergent. For example, if $X$ is a Banach space with a boundedly complete Schauder basis $\left\{e_{n}\right\}_{n}$, and corresponding biorthogonal functionals $\left\{f_{n}\right\}_{n} \subset X^{*}$, then for $Y:=$ the closed linear span of $\left\{f_{n}\right\}_{n}$ in $X^{*}, Y^{*}$ is isomorphic to $X$ and every a.f.p.s. in $X$ has a subsequence that is $\sigma(X, Y)$-convergent. Therefore, we will subsequently assume that approximate fixed point sequences in bounded subsets of $X$ are $w^{*}$-convergent.

Using Cantor's theorem (see [14] or [11, Lemma 1]), the above argument lets us deduce that if $T: C \rightarrow C$ is a fixed point free nonexpansive mapping, there exist some $a>0$ and a closed convex $T$-invariant subset, denoted again by $C$, such that $\lim \sup _{n}\left\|y_{n}-y\right\|>a$ whenever $\left(y_{n}\right) \subset C$ is an a.f.p.s. and $y=w^{*}-\lim y_{n}$.

Note that from Definition 2.1 it is not difficult to check the following [2]:

Lemma 2.5. A norm $\||\cdot|\|$ in a Banach space $X$ with a Schauder basis $\left\{e_{n}\right\}_{n}$ is sequentially separating if and only if $\lim _{k} S_{k}(X,\||\cdot|\|)=1$, with

$$
S_{k}(X,|\|\cdot\||):=\sup \left\{\frac{\left\||| x||\left|+\lim \sup _{n}\right|\right\| x_{n} \| \mid}{\lim \sup _{n}|| x+x_{n}|| \mid}\right\},
$$

where the supremum is taken over all vectors $x \in X$ with $k \leq x$ and all block basic sequences of $\left\{e_{n}\right\}_{n}$.

If we fix the norm $\||\cdot|\|$ in the Banach space $X$, we will use $S_{k}$ to denote $S_{k}(X,\||\cdot|\|)$.

Lemma 2.6. Let $(X,|| \cdot|| \mid)$ be a Banach space with a boundedly complete Schauder basis $\left\{e_{n}\right\}_{n}$ such that $\||\cdot|\|$ is premonotone and sequentially separating. The following holds: if $\left(x_{n}\right),\left(y_{n}\right)$ are two sequences in $X$ that are $w^{*}$-convergent to $x$ and $y$ respectively, then

$$
\limsup _{m} \limsup _{n}||\left|x_{n}-y_{m}\right|\left\|\geq \limsup _{n}||\left|x_{n}-x\right|\right\|+\underset{m}{\limsup }\left|\left\|y_{m}-y\right\|\right| .
$$

Proof. Let $k \in \mathbb{N}$ and $\delta>0$ be given. Choose a subsequence $\left(x_{n_{\ell}}\right)$ of $\left(x_{n}\right)$ such that

$$
\underset{n}{\limsup }\left\|\mid Q_{k}\left(x_{n}-x\right)\right\|\left\|=\lim _{\ell}\right\| Q_{k}\left(x_{n_{\ell}}-x\right)\|\| .
$$

Fix $m \in \mathbb{N}$. Then

$$
\begin{aligned}
\limsup _{n}\left\|x_{n}-y_{m} \mid\right\| & \geq \limsup _{n}\left\|\mid Q_{k}\left(x_{n}-y_{m}\right)\right\| \| \\
& =\limsup _{n}\left\|\left|Q_{k}\left(x_{n}-x\right)-Q_{k}\left(y_{m}-x\right)\right|\right\| \\
& \geq \limsup _{\ell}\left\|\mid Q_{k}\left(x_{n_{\ell}}-x\right)-Q_{k}\left(y_{m}-x\right)\right\| \| .
\end{aligned}
$$


By the Bessaga-Pełczynski Selection Principle (see, for example, [1, p. 14]), passing to a further subsequence if necessary, we may choose a block basic sequence $\left(u_{\ell}\right)$ of $\left(e_{n}\right)$ such that $Q_{k}\left(u_{\ell}\right)=u_{\ell}$ and $\left\|\left|u_{\ell}-Q_{k}\left(x_{n_{\ell}}-x\right) \|\right|<\delta\right.$. Then

$$
\begin{aligned}
& \limsup _{n}||\left|x_{n}-y_{m}\right|\left\|\geq \underset{\ell}{\lim \sup _{\ell}}||\left|u_{\ell}-Q_{k}\left(y_{m}-x\right) \|\right|-\delta\right. \\
& \geq \frac{1}{S_{k}}\left(\left\|Q_{k}\left(y_{m}-x\right)\right\|\left\|+\underset{\ell}{\lim \sup _{\ell}}\right\|\left|u_{\ell} \|\right|\right)-\delta \\
& \geq \frac{1}{S_{k}}\left(\left\|\left|Q_{k}\left(y_{m}-x\right)\left\|\left|+\lim _{\ell}\right|\right\| Q_{k}\left(x_{n_{\ell}}-x\right)\right|\right\|\right)-2 \delta
\end{aligned}
$$

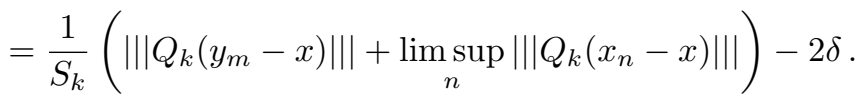

Letting $m$ tend to $\infty$; noting that $S_{k} \geq 1$; and using a perturbation argument similar to the one above then yields:

$$
\begin{aligned}
& \limsup _{m} \limsup _{n}\left\|\left|x_{n}-y_{m} \|\right| \geq \frac{1}{S_{k}}\left(\limsup _{m}\left\|Q_{k}\left(y_{m}-x\right)|\|+\underset{n}{\lim \sup }\|| Q_{k}\left(x_{n}-x\right) \mid\right\|\right)-2 \delta\right. \\
& =\frac{1}{S_{k}}\left(\operatorname { l i m s u p } _ { n } \left\|\left|x_{n}-x\|\mid+\underset{m}{\limsup }\| Q_{k}\left(y_{m}-y\right)+Q_{k}(y-x)\|\|\right)-2 \delta\right.\right. \\
& \geq \frac{1}{S_{k}} \limsup _{n}\left\|\mid x_{n}-x\right\| \|+\frac{1}{S_{k}^{2}}\left(\left\|\left|Q_{k}(y-x)\|\|+\limsup _{m}\left\|\mid Q_{k}\left(y_{m}-y\right)\right\| \|\right)-4 \delta\right.\right. \\
& \geq \frac{1}{S_{k}} \limsup _{n}\left\|\left|x_{n}-x\|\|+\frac{1}{S_{k}^{2}} \limsup _{m}\left\|\mid Q_{k}\left(y_{m}-y\right)\right\| \|-4 \delta\right.\right. \\
& =\frac{1}{S_{k}} \limsup _{n}\left\|\left|x_{n}-x\|\|+\frac{1}{S_{k}^{2}} \limsup _{m}\left\|\mid y_{m}-y\right\| \|-4 \delta .\right.\right.
\end{aligned}
$$

In the above calculation, we used the fact that $\lim \sup _{n}||\left|Q_{k}\left(x_{n}-x\right)\right|||=\lim \sup _{n}|| \mid x_{n}-$ $x \mid \|$ and $\lim \sup _{m}||\left|Q_{k}\left(y_{m}-y\right)\right|\left\|=\lim \sup _{m}\right\|\left|y_{m}-y \|\right|$ for every $k \in \mathbb{N}$.

Since the above inequalities hold for every $k \in \mathbb{N}$, letting $k$ tend to infinity gives

$\limsup \lim \sup \left|\left\|x_{n}-y_{m}|\|\geq \limsup \|| x_{n}-x|\|+\lim \sup \|| y_{m}-y \mid\right\|-4 \delta\right.$, for every $\delta>0$. Since $\delta>0$ is arbitrary, we obtain the desired inequality.

Theorem 2.7. Let $X$ be a Banach space with a boundedly complete Schauder basis and let $|\|\cdot\|| \mid$ be a near-infinity concentrated (n.i.c.) norm on $X$. Then $(X,\||\cdot|\|)$ has the FPP, that is, every nonexpansive self-map on a closed bounded convex subset of $X$ has a fixed point.

Proof. Assume, to the contrary, that there exists a closed bounded convex subset $C$ of $X$ and $T: C \rightarrow C$ a nonexpansive mapping such that

$$
b=\inf \left\{\lim \sup \|\| y_{n}-y\|\|:\left(y_{n}\right) \subset C \text { is an a.f.p.s. and } y_{n} \stackrel{w^{*}}{\rightarrow} y\right\}>0 .
$$

Without loss of generality we can assume that $b=1$. We proceed as follows.

Fix some $0<\epsilon_{1}<\min \left\{\frac{1}{4}\left(1-\frac{M+1}{2}\right), \frac{1}{10}\left(R_{0}-5\right)\right\}$, where $M \in[0,1)$ and $R_{0}>5$ are the constants given by condition (3) in Definition 2.2 .

Consider an a.f.p.s. $\left(x_{n}\right)$ in $C$ such that $x_{n} \stackrel{w^{*}}{\rightarrow} x_{0} \in X$ and $\lim \sup _{n}\left\|\left|x_{n}-x_{0} \|\right|<\right.$ $1+\epsilon_{1}$. Again, without loss of generality, we can assume that $x_{0}=0$ so that $\lim \sup _{n}\left\|\left|x_{n}\right|\right\|<1+\epsilon_{1}$. Define the set

$$
D:=\left\{z \in C: \limsup _{n}\left|\left\|x_{n}-z \mid\right\| \leq 2\left(1+\epsilon_{1}\right)\right\} .\right.
$$


Then $D$ is a closed convex $T$-invariant subset of $C$. Moreover, using the triangle inequality,

$$
\limsup \limsup _{n}||\left|x_{n}-x_{m}\right|\|\leq 2 \limsup \||| x_{n} \mid \|<2\left(1+\epsilon_{1}\right)
$$

so $D$ is not empty and we can assume that $x_{n} \in D$ for $n$ large enough. Define

$$
c:=\inf \left\{\limsup _{n}\left\|\mid y_{n}-y\right\| \|:\left(y_{n}\right) \subset D \text { is an a.f.p.s. and } y_{n} \stackrel{w^{*}}{\rightarrow} y\right\} .
$$

Notice that $1 \leq c$.

To simplify the notation, we define

$$
A^{*}(D):=\left\{y \in X: \exists\left(y_{n}\right) \subset D \text { an a.f.p.s. such that } w^{*}-\lim _{n} y_{n}=y\right\} .
$$

We now prove that $\sup _{y \in A^{*}(D)}\|y\| \mid \leq 4+4 \epsilon_{1}$ :

Indeed, let $\left(y_{n}\right) \subset D$ be an a.f.p.s. with $w^{*}-\lim y_{n}=y$. In particular, $\lim \sup _{n} \| \mid x_{n}-$ $y_{m} \| \mid \leq 2\left(1+\epsilon_{1}\right)$ for every $m \in \mathbb{N}$. Using the triangle inequality and Lemma 2.6 (with $x=0$ ):

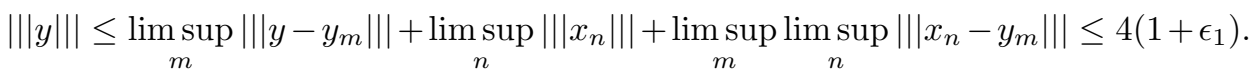

Next we show that $\sup _{y \in A^{*}(D)}\left\|Q_{k}(y)\right\| \leq \mu_{k}:=2 S_{k}^{2}\left(1+\epsilon_{1}\right)-S_{k}-1$ for every $k \in \mathbb{N}$. Using the proof of Lemma 2.6 we deduce that:

$$
\begin{aligned}
& 2\left(1+\epsilon_{1}\right) \geq \limsup _{m} \limsup _{n}\left|\left\|x_{n}-y_{m} \mid\right\| \geq\right. \\
& \frac{1}{S_{k}} \limsup _{n}\|\| x_{n}\|\|+\frac{1}{S_{k}^{2}}\left[\limsup _{m}\left\|y_{m}-y\right\|\|+\| Q_{k}(y)\|\|\right] \geq \frac{1}{S_{k}}+\frac{1}{S_{k}^{2}}\left[1+\left\|Q_{k}(y)\right\| \|\right],
\end{aligned}
$$
which implies that

$$
||\left|Q_{k}(y)\right||| \leq 2 S_{k}^{2}\left(1+\epsilon_{1}\right)-S_{k}-1
$$

for all $y \in A^{*}(D)$.

Choose $x:=x_{\nu}$ with $\nu \in \mathbb{N}$ large enough so that $x \in D$ and $\||x|\|<1+\epsilon_{1}$. Since the norm satisfies condition (1) in Definition 2.2 , we know that $\lim _{k} S_{k}=1$ and therefore $\lim _{k} \mu_{k}=2 \epsilon_{1}$. Take $k_{1} \in \mathbb{N}$ so that

$$
\left\|Q_{k}(x)\right\| \mid<\epsilon_{1}, \quad \text { and } \mu_{k}<3 \epsilon_{1}
$$

if $k \geq k_{1}$. In particular, this implies that

$$
\left\|\left|Q _ { k _ { 1 } } ( y - x ) \left\|\left|\leq\left\|\left|Q_{k_{1}}(y)\|||+\| Q_{k_{1}}(x) \|\right| \mid \leq 3 \epsilon_{1}+\epsilon_{1}=4 \epsilon_{1}<1,\right.\right.\right.\right.\right.
$$

and

$$
\begin{aligned}
\left\|P_{k_{1}}(y-x)\right\| \| & \leq\left\|\left|x-y\left\|\left|+\left\|Q_{k_{1}}(y-x) \mid\right\|\right.\right.\right.\right. \\
& \leq\left\||x\|||+\| y \||+4 \epsilon_{1}\right. \\
& \leq 1+\epsilon_{1}+4+4 \epsilon_{1}+4 \epsilon_{1}=5+9 \epsilon_{1}<R_{0}
\end{aligned}
$$

for every $y \in A^{*}(D)$.

Given $k_{1} \in \mathbb{N}$ as before, there exists a corresponding function $F(\lambda):=F_{k_{1}}(\lambda)$ satisfying property (3) in Definition 2.2. Since $\lim _{\lambda \rightarrow 0^{+}} \frac{F(\lambda)}{\lambda} \leq \frac{M}{R_{0}}$, take $\lambda \in(0,1)$ such that

which implies that

$$
\frac{F(\lambda)}{\lambda}<\frac{M+1}{2 R_{0}}<\frac{1-4 \epsilon_{1}}{R_{0}} \leq \frac{c-4 \epsilon_{1}}{R_{0}}
$$

Now, choose $\epsilon_{2}>0$ with

$$
(2-\lambda) c+F(\lambda) R_{0}+\lambda 4 \epsilon_{1}<2 c .
$$

$$
(2-\lambda)\left(c+\epsilon_{2}\right)+F(\lambda) R_{0}+\lambda 4 \epsilon_{1}<2 c .
$$


Choose $\left(y_{n}\right) \subset D$ an a.f.p.s. with $w^{*}-\lim _{n} y_{n}=y$ and such that

$$
\limsup \left\|\left|y_{n}-y \|\right| \leq c+\epsilon_{2}\right. \text {. }
$$

By passing to a subsequence, we may also suppose that

$$
\liminf _{n}\|\| y_{n}-y\|\|=\limsup _{n}\left\|\left|y_{n}-y \|\right| \geq c \geq 1\right. \text {. }
$$

Notice that, for every $m \in \mathbb{N}$, the vectors $(1-\lambda) y_{m}+\lambda x \in D$. We claim that

$(* *) \quad \limsup _{m} \limsup _{n}\left\|y_{n}-\left[(1-\lambda) y_{m}+\lambda x\right]\right\| \mid<2 c$.

Assume that $(* *)$ holds. Then we can find some $m \in \mathbb{N}$ such that

$$
\limsup _{n}\left\|y_{n}-\left[(1-\lambda) y_{m}+\lambda x\right]\right\| \mid<2 c .
$$

This implies that for some $r \in(0,2 c)$ the set

$$
G:=\left\{z \in D: \limsup _{n}\left\|y_{n}-z \mid\right\| \leq r\right\}
$$

is a nonempty closed convex $T$-invariant subset of $D$, and therefore it contains an a.f.p.s. $\left(z_{s}\right)$, which tends to some $z \in X$ with respect to the $w^{*}$-topology. In this case, using the definition of $c$, Lemma 2.6, and that each $z_{s} \in G$, we have

$$
2 c \leq \limsup _{s}\left\|\left|z_{s}-z\right|\right\|+\limsup _{n}|| y_{n}-y\|\| \leq \limsup _{s} \limsup _{n}\left\|\left|y_{n}-z_{s}\right|\right\| \leq r,
$$

which is a contradiction.

We finish by proving the claim $(* *)$. Noting that $\liminf _{n}\left\|\left|y_{n}-y \|\right| \geq 1\right.$ and by property (3) in Definition 2.2, we have:

$$
\begin{aligned}
\lim \sup _{n} \mid\left\|\left(y_{n}-y\right)+\lambda P_{k_{1}}(y-x)\right\| \| & \leq \limsup _{n}\left|\left\|y_{n}-y\right\|\|+F(\lambda) \mid\| P_{k_{1}}(y-x)\|\|\right. \\
& \leq c+\epsilon_{2}+F(\lambda) R_{0} .
\end{aligned}
$$

Therefore,

$$
\begin{aligned}
& \lim \sup _{m} \lim \sup _{n}|| y_{n}-\left[(1-\lambda) y_{m}+\lambda x\right] \| \mid \\
& =\lim \sup _{m} \lim \sup _{n}\left\|\left|y_{n}-y+y-(1-\lambda) y_{m}-\lambda x \|\right|\right. \\
& =\lim \sup _{m} \lim \sup _{n}\|\| y_{n}-y+(1-\lambda) y+\lambda y-(1-\lambda) y_{m}-\lambda x\|\| \\
& \leq \lim \sup _{m} \lim \sup _{n}\left[(1-\lambda)\left\|\left|y_{m}-y \|\right||+|\left|\left(y_{n}-y\right)+\lambda(y-x)\right|||\right]\right. \\
& \leq(1-\lambda) \lim \sup _{m}\left|\left\|y_{m}-y\right\|\left\|+\lim \sup _{n}\right\|\right|\left(y_{n}-y\right)+\lambda P_{k_{1}}(y-x)\left\|\left|+\lambda\left\|\mid Q_{k_{1}}(y-x)\right\| \|\right.\right. \\
& \leq(1-\lambda)\left(c+\epsilon_{2}\right)+c+\epsilon_{2}+F(\lambda) R_{0}+\lambda 4 \epsilon_{1} \\
& \leq(2-\lambda)\left(c+\epsilon_{2}\right)+F(\lambda) R_{0}+\lambda 4 \epsilon_{1} \\
& <2 c,
\end{aligned}
$$

which proves $(* *)$, and completes the proof of the theorem.

\section{Norms with the Fixed Point Property}

Throughout this section, we will study several examples of norms which are near-infinity concentrated norms and therefore they satisfy the FPP according to Theorem 2.7. As a particular case of a more general result, we will deduce that $\left(\ell_{1}, \nu_{p}(\cdot)\right)$ has the FPP whenever $\nu_{p}(\cdot)$ is a renorming of $\ell_{1}$.

We will start by proving that P.K. Lin's norm is an example of a near-infinity concentrated norm. We will deduce this assertion from the following lemma.

Lemma 3.1. Let $(X,|\cdot|)$ be a Banach space with a Schauder basis and assume that $|\cdot|$ satisfies properties (1) and (2) in Definition 2.1. If $\left(\gamma_{k}\right)$ is a nondecreasing sequence in $(0,1)$ converging to 1 , then the norm defined as

$$
|x|_{1}:=\sup _{k} \gamma_{k}\left|Q_{k}(x)\right|, \text { for all } x \in X
$$


is a near-infinity concentrated norm on $X$ that is equivalent to $|\cdot|$.

Proof. Notice that $\gamma_{k}\left|Q_{k}(x)\right| \leq\left|Q_{k}(x)\right|_{1} \leq\left|Q_{k}(x)\right|$ for every $k \in \mathbb{N}$ and $x \in X$, which implies that $|\cdot|_{1}$ is a sequentially separating norm whenever $|\cdot|$ satisfies the same property. It is also easy to check that $|\cdot|_{1}$ satisfies $(2)$ in Definition 2.2 . It remains to prove condition (3). Fix some $k \in \mathbb{N}$ and $R>0$. Let $\left(x_{n}\right)$ be a bounded pointwise-null sequence in $X$ with $\lim \inf _{n}|x|_{1} \geq 1$. Without loss of generality we can assume that $Q_{l}\left(x_{n}\right)=Q_{k}\left(x_{n}\right)$ for every $l \leq k$. Moreover, it is not difficult to check that $\lim \sup _{n}\left|x_{n}\right|=\limsup _{n}\left|x_{n}\right|_{1}$. For every $z \in X$ with $Q_{k}(z)=0$, $|z|_{1} \leq R$ and for every $\lambda>0$ we have

$$
\begin{aligned}
\left|x_{n}+\lambda z\right|_{1} & =\sup _{l} \gamma_{l}\left|Q_{l}\left(x_{n}+\lambda z\right)\right|=\sup _{l} \gamma_{l}\left|Q_{l}\left(x_{n}\right)+\lambda Q_{l}(z)\right| \\
& =\max \left\{\max _{1 \leq l \leq k-1} \gamma_{l}\left|Q_{l}\left(x_{n}\right)+\lambda Q_{l}(z)\right|, \sup _{l \geq k} \gamma_{l}\left|Q_{l}\left(x_{n}\right)\right|\right\} \\
& =\max \left\{\max _{1 \leq l \leq k-1} \gamma_{l}\left|Q_{l}\left(x_{n}\right)+\lambda Q_{l}(z)\right|,\left|x_{n}\right|_{1}\right\} \\
& \leq \max \left\{\gamma_{k-1}\left|x_{n}\right|+\lambda|z|_{1},\left|x_{n}\right|_{1}\right\}
\end{aligned}
$$

Taking limits when $n$ goes to infinity:

$$
\limsup _{n}\left|x_{n}+\lambda z\right|_{1} \leq \max \left\{\gamma_{k-1} \limsup _{n}\left|x_{n}\right|_{1}+\lambda|z|_{1}, \limsup _{n}\left|x_{n}\right|_{1}\right\} .
$$

From above, $\lim \sup _{n}\left|x_{n}\right|_{1} \geq 1$ and $|z|_{1} \leq R$; and so

$$
\limsup _{n}\left|x_{n}+\lambda z\right|_{1} \leq \limsup _{n}\left|x_{n}\right|_{1}+F_{k}(\lambda)|z|_{1}
$$

where $F_{k}(\lambda):=0$ if $\lambda \leq\left(1-\gamma_{k-1}\right) / R$, and $F_{k}(\lambda):=\lambda$ otherwise. Taking $M=0$ and any $R>5$ in Definition $2.2(3)$, we see that $|\cdot|_{1}$ is a near infinity concentrated norm.

If we let $|\cdot|:=\|\cdot\|_{1}$ in $\ell_{1}$ we obtain that $|\cdot|_{1}$ coincides with P.K. Lin's norm $\|\left.|\cdot|\right|_{L}$. Moreover, given a norm $|\cdot|_{0}:=|\cdot|$ satisfying (1) and (2) in Definition 2.2 and defining in a recursive way the equivalent norms

$$
|\cdot|_{n}=\sup _{k} \gamma_{k}\left|Q_{k}(\cdot)\right|_{n-1}
$$

for every $n \in \mathbb{N}$, we can construct sequences of near-infinity concentrated norms. All of these norms $|\cdot|_{n}(n \geq 1)$ satisfy the FPP when the basis is boundedly complete, according to Theorem 2.7 .

Lemma 3.2. Assume that $\left(p_{n}\right) \subset(1,+\infty)$ is a nonincreasing sequence with $\lim _{n} p_{n}=$ 1. Then the norm $\nu_{p}(\cdot)$ is a near-infinity concentrated norm in the Banach space $X$, defined as the completion of $c_{00}$ with the norm $\nu_{p}(\cdot)$.

Proof. Let us start by proving that $\nu_{p}(\cdot)$ is a sequentially separating norm, that is, $\nu_{p}(\cdot)$ satisfies property (1) in Definition 2.2. By Lemma 2.5, it suffices to check that $\lim _{k} S_{k}\left(X, \nu_{p}(\cdot)\right)=1$.

Fix $k \in \mathbb{N}$. First note that if $x=\sum_{i=k}^{l} x(i) e_{i}$ with $k \leq l$ and $l<y$ then

$$
\nu_{p}(x+y)=\nu_{p}\left(\sum_{i=k}^{l} x(i) e_{i}+\nu_{p}(y) e_{l+1}\right) .
$$

On the other hand, it is not difficult to check that if $1<p \leq q$ and $a, b, c \geq 0$ then:

$$
\begin{gathered}
\left\|\left(a,\|(b, c)\|_{p}\right)\right\|_{q} \geq\left\|\left(a,\|(b, c)\|_{q}\right)\right\|_{q}=\left(a^{q}+\|(b, c)\|_{q}^{q}\right)^{1 / q}=\left(a^{q}+b^{q}+c^{q}\right)^{1 / q}= \\
=\left\|\left(\|(a, b)\|_{q}, c\right)\right\|_{q} .
\end{gathered}
$$


Using now the definition of the norm $\nu_{p}(\cdot)$ and taking into account that

$$
\cdots p_{n} \leq p_{n-1} \leq \cdots p_{2} \leq p_{1}
$$

it can recursively be checked that for $x=\sum_{i=k}^{l} x(i) e_{i}$ with $k \leq l$ and $l<y$

$$
\begin{aligned}
\nu_{p}(x+y) & =\nu_{p}\left(\sum_{i=k}^{l} x(i) e_{i}+\nu_{p}(y) e_{l+1}\right) \\
& \geq\left\|\left(\nu_{p}(x), \nu_{p}(y)\right)\right\|_{p_{k}} \\
& \geq C_{k}\left(\nu_{p}(x)+\nu_{p}(y)\right)
\end{aligned}
$$

where $C_{k}:=2^{-1+1 / p_{k}}$.

Let $\left(x_{n}\right)$ be a block basic sequence in $X$ and let $x \in X$ with $Q_{k}(x)=x$. If $\epsilon>0$ is given, choose $k^{\prime}>k$ such that

$$
\nu_{p}\left(Q_{k^{\prime}}(x)\right)<\epsilon\left(\nu_{p}(x)+\underset{n}{\lim \sup } \nu_{p}\left(x_{n}\right)\right) .
$$

Without loss of generality, we can choose $n$ large enough so that $k^{\prime}<x_{n}$. Applying $(\dagger)$ to the vectors $x_{0}:=x-Q_{k^{\prime}}(x), x_{1}:=Q_{k^{\prime}}(x)$, and $x_{n}$, we obtain:

$$
\begin{aligned}
\limsup \nu_{p}\left(x+x_{n}\right) & \geq \limsup _{n} \nu_{p}\left(x_{0}+x_{n}\right)-\nu_{p}\left(x_{1}\right) \\
& \geq \limsup _{n} C_{k}\left(\nu_{p}\left(x_{0}\right)+\nu_{p}\left(x_{n}\right)\right)-\nu_{p}\left(x_{1}\right) \\
& \geq C_{k}\left(\nu_{p}(x)-\nu_{p}\left(x_{1}\right)+\lim \sup _{n} \nu_{p}\left(x_{n}\right)\right)-\nu_{p}\left(x_{1}\right) \\
& =C_{k}\left(\nu_{p}(x)+\lim \sup _{n} \nu_{p}\left(x_{n}\right)\right)-\left(C_{k}+1\right) \nu_{p}\left(x_{1}\right) \\
& \geq C_{k}\left(\nu_{p}(x)+\lim \sup _{n} \nu_{p}\left(x_{n}\right)\right)-\left(C_{k}+1\right) \epsilon\left(\nu_{p}(x)+\lim \sup _{n} \nu_{p}\left(x_{n}\right)\right) \\
& =\left(C_{k}-\epsilon\left(C_{k}+1\right)\right)\left(\nu_{p}(x)+\lim \sup _{n} \nu_{p}\left(x_{n}\right)\right)
\end{aligned}
$$

Since $\epsilon>0$ is arbitrary,

$$
\limsup _{n} \nu_{p}\left(x+x_{n}\right) \geq C_{k}\left(\nu_{p}(x)+\limsup _{n} \nu_{p}\left(x_{n}\right)\right) .
$$

Then, by the definition of the coefficient $S_{k}\left(X, \nu_{p}(\cdot)\right)$, we deduce that

$$
S_{k}\left(X, \nu_{p}(\cdot)\right) \leq \frac{1}{C_{k}}
$$

Taking limits as $k$ goes to infinity and using Lemma 2.5 shows that $\nu_{p}(\cdot)$ is a sequentially separating norm.

It is clear that $\nu_{p}(\cdot)$ satisfies (2) in Definition 2.2. Therefore, it remains to prove (3) in Definition 2.2.

Fix $k \in \mathbb{N}$. Consider the equivalent finite dimensional Banach spaces $\left(\mathbb{R}^{k},\|\cdot\|_{p_{k}}\right)$ and $\left(\mathbb{R}^{k}, \nu_{p}(\cdot)\right)$. Take some constant $L_{k}>0$ such that $\|x\|_{p_{k}} \leq L_{k} \nu_{p}(x)$ for every $x \in \mathbb{R}^{k}$.

Let $\left(x_{n}\right)$ be a bounded pointwise-null sequence with $\liminf \operatorname{in}_{n} \nu_{p}\left(x_{n}\right) \geq 1$, and $z \in X$ with $Q_{k}(z)=0$ and $\nu_{p}(z) \leq R$ for some $R>0$. We can assume, without loss of generality, that $P_{k}\left(x_{n}\right)=0$ for every $n \in \mathbb{N}$.

Having in mind that $\left(p_{n}\right)_{n}$ is a nonincreasing sequence, it is not difficult to check that for all $\lambda \in(0,+\infty)$,

$$
\begin{aligned}
\nu_{p}\left(x_{n}+\lambda z\right) & \leq\left(\lambda^{p_{k}}|z(1)|^{p_{k}}+\cdots \lambda^{p_{k}}|z(k-1)|^{p_{k}}+\nu_{p}\left(x_{n}\right)^{p_{k}}\right)^{1 / p_{k}} \\
& =\nu_{p}\left(x_{n}\right)\left[\frac{\lambda^{p_{k}}}{\nu_{p}\left(x_{n}\right)^{p_{k}}}\left(|z(1)|^{p_{k}}+\cdots|z(k-1)|^{p_{k}}\right)+1\right]^{1 / p_{k}} \\
& =\nu_{p}\left(x_{n}\right)\left[\frac{\lambda^{p_{k}}}{\nu_{p}\left(x_{n}\right)^{p_{k}}}\|z\|_{p_{k}}^{p_{k}}+1\right]^{1 / p_{k}} .
\end{aligned}
$$


It is easy to check that $(1+v)^{\alpha} \leq 1+\alpha v$ if $0<v$ and $0<\alpha<1$. Therefore

$$
\begin{aligned}
\nu_{p}\left(x_{n}+\lambda z\right) & \leq \nu_{p}\left(x_{n}\right)\left[\frac{1}{p_{k}} \frac{\lambda^{p_{k}}}{\nu_{p}\left(x_{n}\right)^{p_{k}}}\|z\|_{p_{k}}^{p_{k}}+1\right] \\
& =\nu_{p}\left(x_{n}\right)+\frac{1}{p_{k}} \frac{\lambda^{p_{k}}}{\nu_{p}\left(x_{n}\right)^{p_{k}-1}}\|z\|_{p_{k}}^{p_{k}} \\
& \leq \nu_{p}\left(x_{n}\right)+\frac{1}{p_{k}} \frac{\lambda^{p_{k}}}{\nu_{p}\left(x_{n}\right)^{p_{k}-1}} L_{k}^{p_{k}} \nu_{p}(z)^{p_{k}} .
\end{aligned}
$$

Then, for every $\lambda>0$,

$$
\begin{aligned}
\limsup _{n} \nu_{p}\left(x_{n}+\lambda z\right) & \leq \limsup _{n} \nu_{p}\left(x_{n}\right)+\frac{1}{p_{k}} \frac{\lambda^{p_{k}}}{\liminf _{n} \nu_{p}\left(x_{n}\right)^{p_{k}-1}} L_{k}^{p_{k}} \nu_{p}(z)^{p_{k}-1} \nu_{p}(z) \\
& \leq \limsup _{n} \nu_{p}\left(x_{n}\right)+\frac{1}{p_{k}} \lambda^{p_{k}} L_{k}^{p_{k}} R^{p_{k}-1} \nu_{p}(z) .
\end{aligned}
$$

Define

$$
F_{k}(\lambda):=\frac{1}{p_{k}} \lambda^{p_{k}} L_{k}^{p_{k}} R^{p_{k}-1},
$$

for every $\lambda>0$. Since, $\lim _{\lambda \rightarrow 0^{+}} F_{k}(\lambda) / \lambda=0$, we can take $M=0$ in property (3) of Definition 2.2 and $\nu_{p}(\cdot)$ is a near-infinity concentrated norm whenever $\lim _{n} p_{n}=1$.

Corollary 3.3. For every nonincreasing sequence $\left(p_{n}\right) \subset(1,+\infty)$ with $\lim _{n} p_{n}=1$, the Banach space $\left(X, \nu_{p}(\cdot)\right)$ satisfies the FPP. In particular, if the sequence of conjugates of $\left(p_{n}\right)$ satisfies that $q_{n} \geq \delta \log n$ for all $n \in \mathbb{N}$ and some $\delta>0$, the norm $\nu_{p}(\cdot)$ is an equivalent norm in $\ell_{1}$ with the FPP.

Remark 3.4. We would like to point out that there exist some norms verifying the FPP which are not near-infinity concentrated. For instance we can consider the Banach space $\ell_{1}$ and the equivalent norm $|x|_{1}=\left.\left\||| P_{A}(x)\right\|\right|_{L}+\left\|\mid P_{B}(x)\right\| \|_{L}$ where $A=\{2 n: n \geq 1\}, B=\{2 n-1: n \geq 1\}, P_{A}, P_{B}$ denote the corresponding projections onto the subspaces $\left[e_{n}: n \in A\right]$ and $\left[e_{n}: n \in B\right]$ respectively and $\||\cdot|\|_{L}$ denotes P.K. Lin's norm as usual. It is easy to check that $\left(\ell_{1},|\cdot|_{1}\right)$ is isometric to the space $\left(\ell_{1},|||\cdot| \|_{L}\right) \oplus_{1}\left(\ell_{1},|||\cdot| \|_{L}\right)$, which has the FPP according to [6]. However, $|\cdot|_{1}$ does not satisfies condition (3) in Definition 2.2.

A similar situation occurs when we consider the $\ell_{1}$-renorming $|x|_{1}=\|x\|_{1}+$ $\||| x \mid\|_{L}$. From [10] we know that $\left(\ell_{1},\|x\|_{1}+\||| x \mid\|_{L}\right)$ verifies the FPP. However, condition (3) in Definition 2.2 also fails in this example.

Nevertheless, by means of Theorem 2.7 , we can obtain a stability result in the following sense.

Theorem 3.5. Let $\||\cdot|||$ be a near-infinity concentrated norm on a Banach space $X$ with a Schauder basis $\left\{e_{n}\right\}_{n}$, and let $\|\cdot\|$ be an equivalent norm satisfying conditions (1) and (2) in Definition 2.2. Then there exists $r_{0}>0$ such that the norm $|\cdot|=\left|\|\cdot|| \mid+r\| \cdot \|\right.$ is also near-infinity concentrated for every $0 \leq r \leq r_{0}$.

Moreover, if $\left\{e_{n}\right\}_{n}$ is boundedly complete, then the spaces $(X,|\cdot|)$ have the FPP for every $0 \leq r \leq r_{0}$.

Proof. Since $\||\cdot|\|$ and $\|\cdot\|$ are equivalent norms, there exist $0<a \leq b$ such that $a|\|x|\|\leq\| x\|\leq b\|||x|\|$ for every $x \in X$. Therefore, for all $x \in X$,

$$
(1+a r)\left|\|x|\|\leq|x| \leq(1+r b)\|||x|\| \text { and }\left(\frac{1}{b}+\frac{r a}{b}\right)\|x\| \leq\right| x \mid \leq\left(\frac{1}{a}+\frac{r b}{a}\right)\|x\| \text {. }
$$

It is easy to check that $|\cdot|$ satisfies conditions (1) and (2). Let us prove (3). By hypotheses, there exists some $R_{0}>5$ and $M \in[0,1)$ satisfying Definition 2.2 .3 for the \|\|$\cdot \|$ norm. Take some $M^{\prime} \in(M, 1)$. Let $\left(x_{n}\right)$ be a bounded pointwise-null sequence with $\liminf \operatorname{in}_{n}\left|x_{n}\right| \geq 1$ and let $z \in X$ with $Q_{k}(z)=0$ and $|z| \leq R_{0}$. In this 
case, $\liminf _{n}||\left|x_{n}\right| \| \geq 1 /(1+r b)$ and ||$|z||| \leq R_{0} /(1+a r)$. Fix $\lambda \in(0, \infty)$. Then there exists a subsequence $\left(x_{n_{j}}\right)_{j \in \mathbb{N}}$ of $\left(x_{n}\right)_{n \in \mathbb{N}}$ such that

$$
\Gamma:=\limsup _{n}\left|x_{n}+\lambda z\right|=\lim _{j}\left|x_{n_{j}}+\lambda z\right| .
$$

Taking further subsequences if necessary, we may assume without loss of generality, that the limits

$$
\lim _{j}\left\||| x_{n_{j}}\right\|\left|, \lim _{j}\left\|x_{n_{j}}\right\|, \lim _{j}\left\|\left|x_{n_{j}}+\lambda z\right|\right\| \text { and } \lim _{j}\left\|x_{n_{j}}+\lambda z\right\|\right.
$$

all exist in $[0, \infty)$. Therefore,

$$
\begin{aligned}
\Gamma= & r \lim _{j}\left\|x_{n_{j}}+\lambda z\right\|+\lim _{j}\left\|\left|x_{n_{j}}+\lambda z\right|\right\| \\
\leq & r \lim _{j}\left\|x_{n_{j}}\right\|+\lambda r\|z\| \\
& \quad+\frac{1}{1+r b} \lim _{j}\|\|(1+r b) x_{n_{j}}+\lambda \frac{1+r b}{1+a r}(1+a r) z \| \mid \\
\leq & r \lim _{j}\left\|x_{n_{j}}\right\|+\lambda r\|z\| \\
& \quad+\limsup _{j}\left|\left\|x_{n_{j}}\left|\left\|+F_{k}\left(\frac{\lambda(1+r b)}{1+a r}\right) \frac{1+a r}{1+r b}\right\| z\right|\right\|\right. \\
= & \lim _{j}\left|x_{n_{j}}\right|+\lambda r\|z\|+F_{k}\left(\frac{\lambda(1+r b)}{1+a r}\right) \frac{1+a r}{1+r b}\|z \mid\| \\
\leq & \limsup _{n}\left|x_{n}\right|+\lambda \frac{r}{\left(\frac{1}{b}+\frac{r a}{b}\right)}|z|+G(\lambda) \frac{1}{1+a r}|z| \\
= & \limsup _{n}\left|x_{n}\right|+\left[\lambda \frac{r}{\left(\frac{1}{b}+\frac{r a}{b}\right)}+G(\lambda) \frac{1}{1+a r}\right]|z|,
\end{aligned}
$$

where $G(\lambda):=F_{k}\left(\lambda \frac{(1+r b)}{(1+a r)}\right)\left(\frac{1+r b}{1+a r}\right)^{-1}$. Define the corresponding " $F_{k}$-type" function for the $|\cdot|$ norm by

$$
F_{k}^{\prime}(\lambda):=\lambda \frac{r}{\left(\frac{1}{b}+\frac{r a}{b}\right)}+G(\lambda) \frac{1}{1+a r}, \text { for all } \lambda \in(0, \infty) .
$$

Since $\lim _{\lambda \rightarrow 0^{+}} G(\lambda) / \lambda \leq \frac{M}{R_{0}}$, we have that

$$
\lim _{\lambda \rightarrow 0^{+}} \frac{F_{k}^{\prime}(\lambda)}{\lambda} \leq \frac{r}{\left(\frac{1}{b}+\frac{r a}{b}\right)}+\frac{1}{1+a r} \frac{M}{R_{0}}:=g(r)
$$

Notice that $\lim _{r \rightarrow 0} g(r)=M / R_{0}$, which implies that there exists some $r_{0}>0$ and $M^{\prime} \in(M, 1)$ (depending on the constants $\left.a, b\right)$ such that for every $0 \leq r \leq r_{0}$, $g(r) \leq M^{\prime} / R_{0}$; and so $\|\mid \cdot\|\|+r\| \cdot \|$ is a near-infinity concentrated norm.

The rest of the theorem follows by applying Theorem 2.7 .

If we proceed as in the previous proof, using the above arguments and the fact that $\lim _{\lambda \rightarrow 0^{+}} \frac{F_{k}(\lambda)}{\lambda}=0$ for both P.K. Lin's norm and the $\nu_{p}(\cdot)$ norm, it is not difficult to check that, in the case where $\nu_{p}(\cdot)$ is equivalent to the $\|\cdot\|_{1}$-norm, $\nu_{p}(\cdot)+\lambda\|\| \cdot\|\|_{L}$ is a renorming in $\ell_{1}$ which is also near-infinity concentrated for every $\lambda>0$. Therefore we can also deduce (see also [2, Section 4]):

Corollary 3.6. Let $\left(p_{n}\right)$ be a nonincreasing sequence in $(1,+\infty)$ such that there exists some $\delta>0$ so that $q_{n} \geq \delta \log n$ for all $n \in \mathbb{N}$. Then $\left(\ell_{1}, \nu_{p}(\cdot)+\lambda \mid\|\cdot\| \|_{L}\right)$ has the FPP for every $\lambda \geq 0$.

Acknowledgment: The authors would like to thank two anonymous reviewers for their constructive and valuable comments, which helped us to improve the quality of this manuscript. 


\section{REFERENCES}

[1] F. Albiac and N.J. Kalton, Topics in Banach Space Theory, Graduate Texts in Mathematics, Springer, 2006.

[2] A. Barrera-Cuevas and M.A. Japón, New families of nonreflexive Banach spaces with the fixed point property, J. Math. Anal. Appl. 425(1) (2015), 349-363.

[3] P.N. Dowling, W.B. Johnson, C.J. Lennard and B. Turett, The optimality of James's distortion theorems, Proc. Amer. Math. Soc. 124 (1) (1997), 167-174.

[4] P.N. Dowling and C.J. Lennard, Every nonreflexive subspace of $L_{1}[0,1]$ fails the fixed point property, Proc. Amer. Math. Soc. 125 (1997), 443-446.

[5] P.N. Dowling, C.J. Lennard and B. Turett, Asymptotically perturbed norms of classical sequence spaces with applications to fixed point theory, Proceedings of Workshop on Fixed Point Theory (Kazimierz Dolny, 1997), Ann. Univ. Mariae Curie-Sklodowska Sect. A 51(2) (1997), 67-98.

[6] P. Dowling, P.K. Lin and B. Turett, Direct sums of renormings of $\ell_{1}$ and the fixed point property, Nonlinear Anal. 73(3) (2010), 591-599.

[7] H. Fetter, P.K. Lin's paper for a larger set to norms equivalent to the $\ell_{1}$ norm, unpublished, 2006.

[8] H. Fetter and B. Gamboa de Buen, Banach spaces with a basis that are hereditarily asymptotically isometric to $\ell_{1}$ and the fixed point property, Nonlinear Anal. 71 (2009), 4598-4608.

[9] K. Goebel and W.A. Kirk, Topics in Metric Fixed Point Theory, Cambridge University Press, 1990.

[10] C.A. Hernández-Linares, M.A. Japón and E. Llorens-Fuster, On the structure of the set of equivalent norms on $\ell_{1}$ with the fixed point property, J. Math. Anal. App. 387 (2012), 645-654.

[11] C.A. Hernández-Linares and M.A. Japón, A renorming in some Banach spaces with applications to fixed point theory, J. of Functional Anal. 250 (10) (2010), 3452-3468.

[12] C.A. Hernández-Linares and M.A. Japón, Rays of equivalent norms with the fixed point property in some nonreflexive Banach spaces, J. Nonlinear Convex Anal. 15(2) (2014), 355377 .

[13] W.A. Kirk and B. Sims, Handbook of Metric Fixed Point Theory, Kluwer Acad. Publ., Dordrecht, 2001.

[14] P.K. Lin, There is an equivalent norm on $\ell_{1}$ that has the fixed point property, Nonlinear Anal. 68 (8) (2008), 2303-2308.

[15] P.K. Lin, Renorming of $\ell_{1}$ and the fixed point property, J. Math. Anal. Appl. 362(2) (2010), 534-541.

Centro de Investigacion en Matematicas (Cimat), Apartado Postal 402, 36000 Guanajuato, Guanajuato, Mexico.

E-mail address: paco@cimat.mx

Department of Mathematics, Miami University, Oxford, OH 45056, USA.

E-mail address: dowlinpn@miamioh.edu

Centro de Investigacion en Matematicas (Cimat), Apartado Postal 402, 36000 Guanajuato, Guanajuato, Mexico.

E-mail address: fetter@cimat.mx

Departamento de Análisis Matemático, Facultad de Matemáticas, Universidad de Sevilla, Tarfia s/n, 41012, Sevilla, Spain.

E-mail address: japon@us.es

Department of Mathematics, University of Pittsburgh, Pittsburgh, PA 15260, USA.

E-mail address: lennard@pitt.edu

CARMA, School of Mathematical and Physical Sciences, University of Newcastle, Callaghan, NSW 2308, Australia.

E-mail address: brailey.sims@newcastle.edu.au

Department of Mathematics, OAkland University, Rochester, Mi 48309, USA.

E-mail address: turett@oakland.edu 